\title{
PYROELECTRIC AND PIEZOELECTRIC PROPERTIES OF GAN-BASED MATERIALS
}

M. S. SHUR*, A. D. BYKHOVSKI*, R. GASKA**

*Department of Electrical, Computer, and Systems Engineering, Rensselaer

Polytechnic Institute, Troy, NY 12180, USA, shurm@ @rpi.edu

**APA Optics, Inc., 2950 N. E. 84th Lane, Blaine, MN 55449, USA

\begin{abstract}
We review pyroelectric and piezoelectric properties of GaN-based materials. Pyroelectric effects in GaN have been studied in two different regimes: (i) uniform sample heating regime and (ii) under applied temperature gradient along the sample. The modeling results show that the pyroelectric coefficient, $P_{v}$, in $\mathrm{GaN}$ (for $c$-axis along the contacts) can reach $7 \times 10^{5} \mathrm{~V} / \mathrm{m}-\mathrm{K}$ (compared to $P_{v}=5 \times 10^{5} \mathrm{~V} / \mathrm{m}-\mathrm{K}$ for the best-known high temperature pyroelectric/piezoelectric material $\mathrm{LiTaO}_{3}$ ). This points to a high potential of $\mathrm{GaN}$-based sensors for high temperature pyroelectronics. Piezoelectric effects strongly affect the performance of electronic and lightemitting devices based on III-N materials. Piezoelectrically induced charge in heterostructures can be as large as 3 to $4 \times 10^{13} \mathrm{~cm}^{-2}$. Hence, strong lattice polarization effects provide unique possibilities for utilizing GaN-based materials in high temperature piezoelectronics and for their applications in pyroelectric detectors.
\end{abstract}

\section{Introduction}

Recent improvements in material quality and contact technology for GaN-based materials system have led to a rapid progress in GaN devices. These devices include blue-green lasers ${ }^{1}$, blue, green, and amber Light Emitting Diodes ${ }^{2}$, Ultraviolet (UV) photodetectors ${ }^{3,4}$, and AlGaN/GaN Heterostructure Field Effect Transistors 5-11. Wide band gap, high peak and saturation velocities, high breakdown voltage and chemical inertness make AlN-GaN-InN based semiconductors an excellent material for solar-blind optoelectronics, and high-power, hightemperature electronics.

GaN-based materials are usually grown in the [0001] direction (when they have the wurtzite crystal structure) and in the [111] direction (when they have the zinc blende crystal structure). These are polar axes, and, therefore, GaN-based materials exhibit strong lattice polarization effects. These effects are uniquely suited for applications in high temperature piezoelectronics and for applications in pyroelectric sensors.

We report on preliminary studies of pyroelectric properties of GaN. Much (if not most) of the work in this area remains to be done, and we expect dramatic improvements in GaN-based pyroelectric sensors still to come. We discuss the piezoelectric properties of III-Nitrides and the application of the concept of "piezoelectric doping" to GaN-based Heterostructure Field Effect Transistors. We consider the issue of spontaneous polarization in wurtzite GaN, AlN, and InN. Finally, we discuss several unresolved issues and make projections of future progress in GaNbased piezoelectronic devices.

\section{Pyroelectricity in gallium nitride thin films}

The pyroelectric materials are capable to generate an electric charge in response to heat flow. Heat sources affect the sample temperature by means of thermal convection, thermal diffusion or radiation. Both the primary pyroelectric effect and the secondary pyroelectric effect (piezoelectric effect caused by temperature induced strain) contribute to the pyroelectric response in GaN. The primary pyroelectric effect is dominant under the condition of a fast heat 
transfer. Such conditions can be implemented, for example, when the sensor is immersed in medium with a small viscosity and /or high flow velocity. In such moving medium (for example, hot gas flow), a GaN-based sensor will generate a voltage response, which is proportional to the heat flow (and, hence, to the gas flow).

Secondary pyroelectricity is linked to strain related to thermal expansion. For example, a difference in thermal expansion coefficients between a substrate and a pyroelectric film produces strain in response to temperature changes, which, in turn, generates an electric charge as a result of the piezoelectric effect.

Our estimates show that the sensitivity of GaN pyroelectric sensors can be significantly improved. The modeling results indicate that the pyroelectric voltage coefficients in $\mathrm{GaN}$ (for $c$ axis along the contacts) can be as high as $7 \times 10^{5} \mathrm{~V} / \mathrm{m}-\mathrm{K}^{12}$ and exceed the $P_{v}=5 \times 10^{5} \mathrm{~V} / \mathrm{m}-\mathrm{K}$ for the best-known high temperature pyroelectric material $\mathrm{LiTaO}_{3}{ }^{13}$.

Therefore, GaN-based sensors should be suitable for high temperature pyroelectronics and piezoelectronics. Moreover, we expect that the pyroelectric effect will be more pronounced in insulating $\mathrm{AlN}$ or in $\mathrm{AlGaN}$ with a large $\mathrm{Al}$ mole fraction..

Low pressure MOCVD was used to fabricate all our samples (for the details of the growth procedure, see ${ }^{14}$ ). The Hall electron concentration was approximately $5 \times 10^{16} \mathrm{~cm}^{-3}$ and the Hall mobility was on the order of $350 \mathrm{~cm}^{2} / \mathrm{V}$-s. A typical sample resistance was close to 2 $\mathrm{k} \Omega$. GaN layers, 3 - $5 \mu \mathrm{m}$ thick, had ohmic contacts on the basal plane.

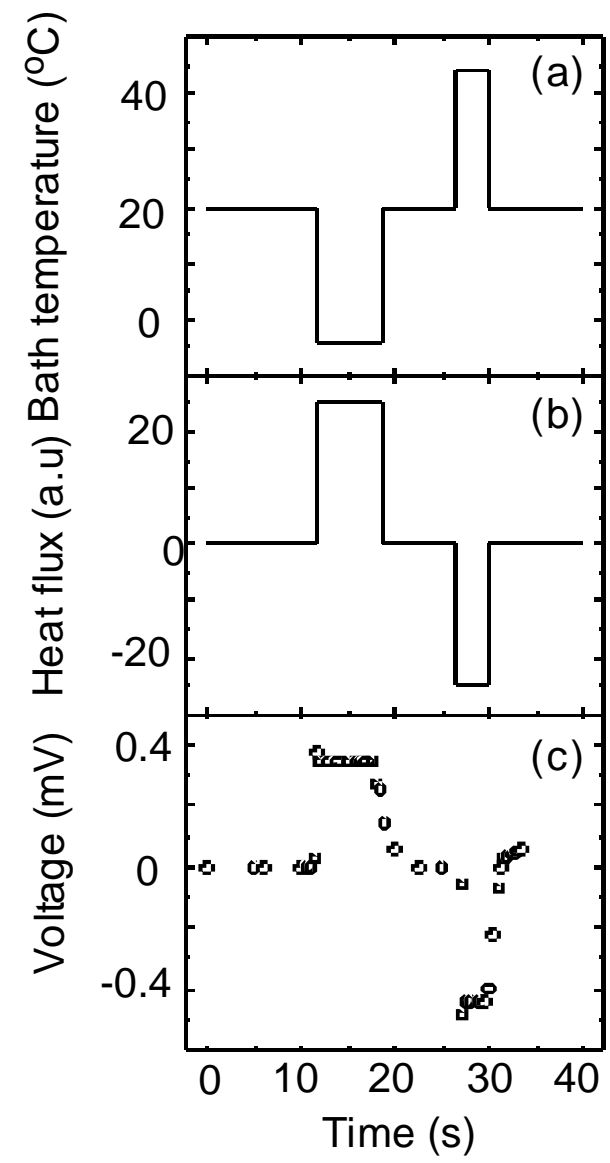

Fig. 1. Pyroelectric voltage versus time for uniform heating and cooling ( after $^{15}$ ).

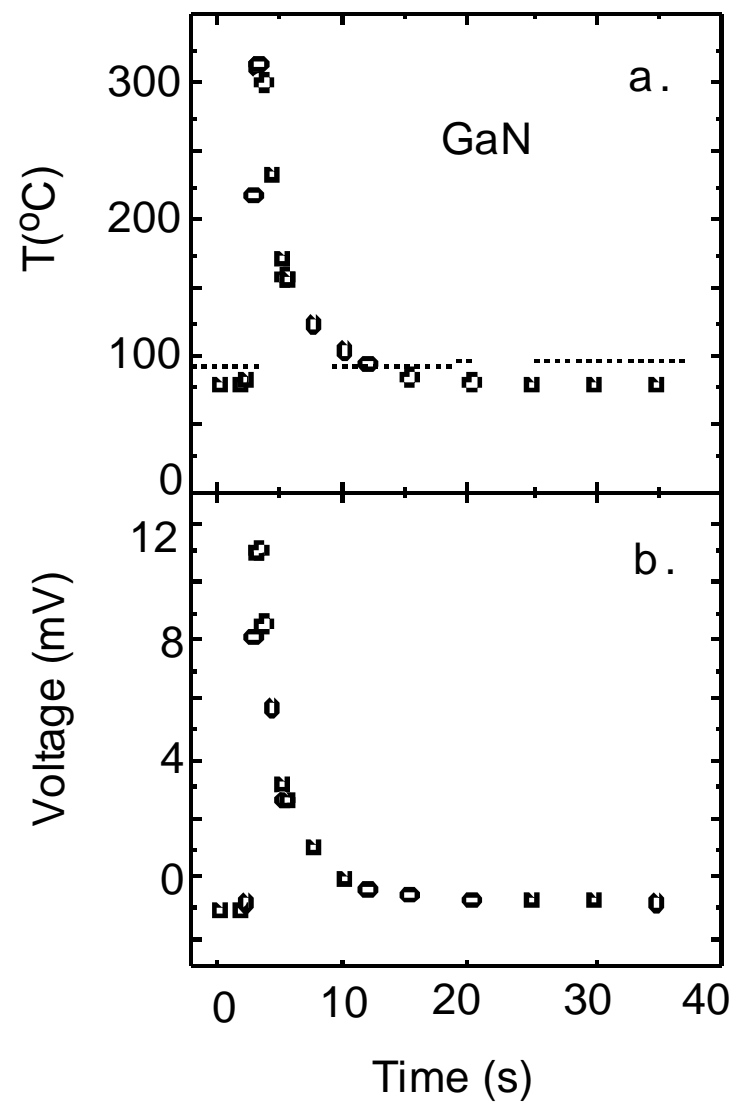

Fig. 2. Dynamics of contact temperature difference (a) and voltage response of $\mathrm{GaN}$ sample under non-uniform heating (b). Constant temperature of "cold" contact $\left(94^{\circ} \mathrm{C}\right)$ is shown by dotted line in Fig. 2 a. 
Pyroelectric properties of GaN were studied in two different regimes: (i) under applied temperature gradient along the sample and (ii) uniform sample heating.

The pyroelectric voltage was measured in the GaN sample subjected to uniform (see Fig. 1) and non-uniform (Fig. 2) heating and cooling. ${ }^{15}$ The time dependence of the voltage response in the GaN sample under applied temperature gradient is shown in Fig. 2 . In these measurements, one contact was kept at a fixed temperature, which varied from $12{ }^{\circ} \mathrm{C}$ to $94{ }^{\circ} \mathrm{C}$. The second contact was heated up to temperatures as high as $350{ }^{\circ} \mathrm{C}$. The ambient temperature changed abruptly and a SmS-based thermoresistor monitored the sample temperature as a function of time. Cold and hot thermal shock experiments in the range from $-100{ }^{\circ} \mathrm{C}$ to $+300{ }^{\circ} \mathrm{C}$ used $96 \%$ ethyl or oil as a thermal bath. In these experiments, the heat transfer was caused mainly by free convection. Since the temperatures were relatively low, the radiative heat transfer was not important. Also, in uniform heating/cooling experiments, when the sample was dipped into the thermal bath, there was no appreciable temperature difference between the contacts, so that the sample did not act as a thermocouple.

Due to a relatively high thermal conductivity of sapphire, the temperature gradients in the sample could be neglected under the uniform heating/cooling conditions, and the lumped system analysis was valid. ${ }^{16}$ The pyroelectric effect in these experiments can be described by the heat transfer equation, the equation of motion of a viscous fluid, and the equation for pyroelectric polarization. Both the sample temperature change, $T(t)-T(0)$, and the heat flow affect the polarization. The temperature change is proportional to the thermal energy stored or dissipated.

In Reference ${ }^{15}$, we described a model, which describes the time variation of the pyroelectric voltage. This model accounts for both primary and secondary pyroelectric effects. In order to compare the pyroelectric properties of $\mathrm{GaN}$ with those of other pyroelectric materials, we estimated the pyroelectric voltage coefficient of $\mathrm{GaN}$ :

$$
P_{v}=\frac{d F}{d T}
$$

where $F$ is the electric field in the sample caused by the pyroelectricity and $T$ is temperature. In order to find the electric field, we solved Poisson's equation taking into account the pyroelectric polarization. For the primary pyroelectricity, such a solution yielded:

$$
P_{v}=\frac{d P_{s}}{\varepsilon_{s} d T} \approx \frac{V\left(t_{o}\right)}{\left(T_{o}-T_{\infty}\right) r_{d}}
$$

Here $P_{s}$ is the spontaneous polarization, $\varepsilon_{s}$ is the static dielectric permittivity of $\mathrm{GaN}, V\left(t_{0}\right)$ is the peak voltage, $T_{0}$ is the initial sample temperature, $T_{\infty}$ is the temperature of the ambiance, and $r_{d}$ is the Debye length. From the experimental data given in Ref. ${ }^{15}$, we estimated $P_{v} \sim 10^{4} \mathrm{~V} / \mathrm{m}-\mathrm{K}$. This value is close to the values of $P_{v}$ for pyroelectric ceramics, such as PZT and $\mathrm{BaTiO}_{3}$. For the contacts along the c-axis, $P_{v}$ might be close to $7 \times 10^{5} \mathrm{~V} / \mathrm{m}-\mathrm{K}$.

Figures 3 and 4 compare the calculated and experimental time dependencies of the pyroelectric voltage and the sample temperature for cooling experiments. The temperature calculated using the free convection heat transfer mechanism (dominant under the conditions of this experiment) is in excellent agreement with the measured data.

Figure 4 shows the time dependence of the pyroelectric voltage for nearly the same temperature differences between the sample and the thermal bath but for the initial heat flows being different by approximately a factor of 3 . These flows were estimated using the thermal boundary layer theory. As can be seen from the figure, the peak voltage increases proportionally to the thermal flux, in agreement with the theory.

Figures 3 and 4 show that the pyroelectric effect in $\mathrm{GaN}$ is a combination of fast and slow responses, in agreement with the proposed model. The fast response (jumps in Figs. 3, 4) is 
caused by the initial thermal flow, which is accounted for by the initial condition in the model described in Reference ${ }^{15}$. In the approximation used in ${ }^{15}$, this response is instant.

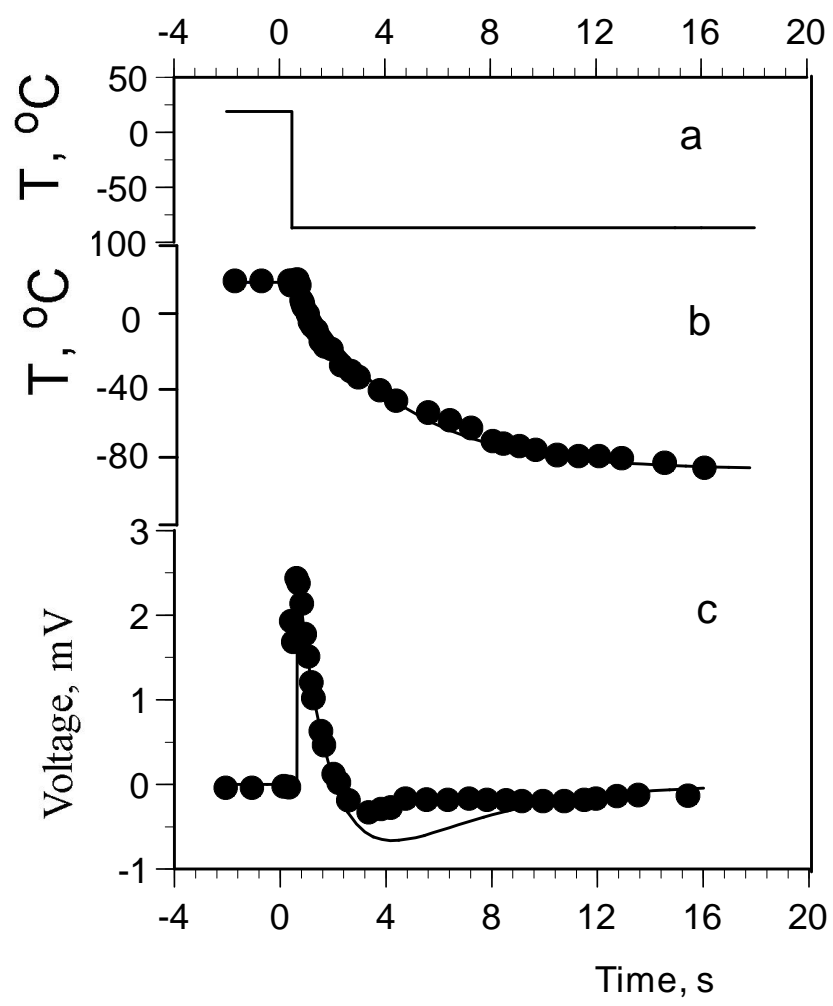

Fig. 3. Bath temperature (a), sample temperature (b), and pyroelectric voltage (c) versus time. Dots are measured data, solid curves are calculated. $\Delta T_{o}=108^{\circ} \mathrm{C} .\left(\right.$ after $\left.^{15}\right)$.

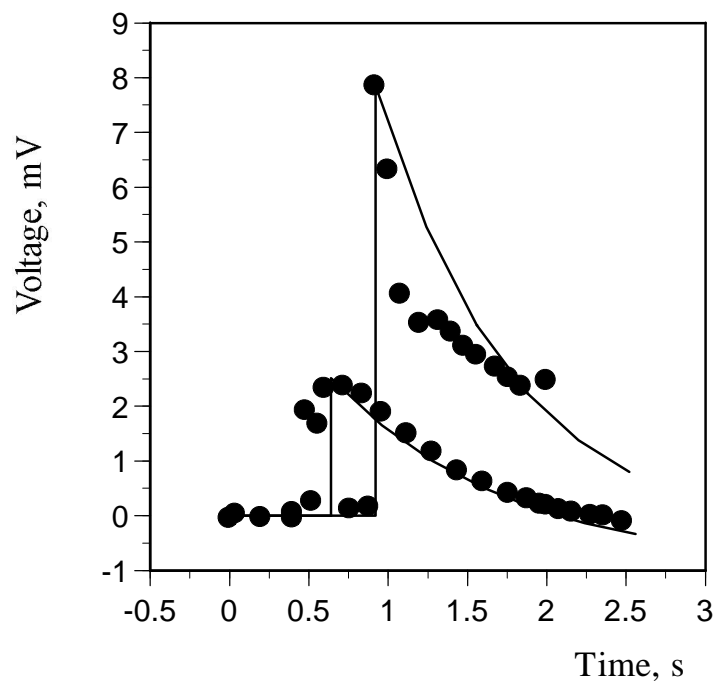

Fig. 4. Pyroelectric voltage as a function of time for conditions similar to that of Fig. 3. Circles are measured data, solid lines are calculated. Upper curve corresponds to initial thermal flux approximately three times larger than for bottom curve. $\Delta T_{o}=-105{ }^{\circ} \mathrm{C}$ (lower curve), $\Delta T_{o}=-$ $99{ }^{\circ} \mathrm{C}$ (upper curve). (After ${ }^{15}$.)

Figures 3 and 4 show that the pyroelectric effect in $\mathrm{GaN}$ is a combination of fast and slow responses, in agreement with the proposed model. The fast response (jumps in Figs. 3, 4) is 
caused by the initial thermal flow, which is accounted for by the initial condition in the model described in Reference ${ }^{15}$. In the approximation used in ${ }^{15}$, this response is instant.

In practice, this time is determined by time that it takes to dip the sample into the thermal bath. The change of the sign of the effect and the minimum on the voltage versus time curve in Fig. 3 is a consequence of the two characteristic relaxation times, $\tau_{T}$, and $\tau_{s}$ associated with the slow response. The first relaxation time, $\tau_{T}$, reflects the rate of the sample cooling due to free convection, and the second relaxation time, $\tau_{s}$, reflects the pyroelectric charge relaxation. The time dependence of the pyroelectric voltage would have been different if free convection were not a dominant heat transfer mechanism $\left(\right.$ see $\left.^{16}\right)$.

Figure 5 compares the experimental and calculated dependencies of the peak voltages on the sample temperature change, $\Delta T_{o}$. The experimental points were measured using cold thermal shock experiments. As can be seen from the figure, the experimental data agree well with the theory ${ }^{15}$. These experiments showed that a GaN sample could be used as a heat flow counter. In order to check this idea further, we subjected the sample first to fast cooling and then to fast heating, within $-4{ }^{\circ} \mathrm{C}$ to $43{ }^{\circ} \mathrm{C}$ temperature range. The absolute value of the temperature difference between the bath and the sample was about the same in each experiment. The sign of the voltage was determined by the direction of the thermal flow (in or out of the sample), and was proportional to the thermal flow (see Fig. 1). ${ }^{15}$

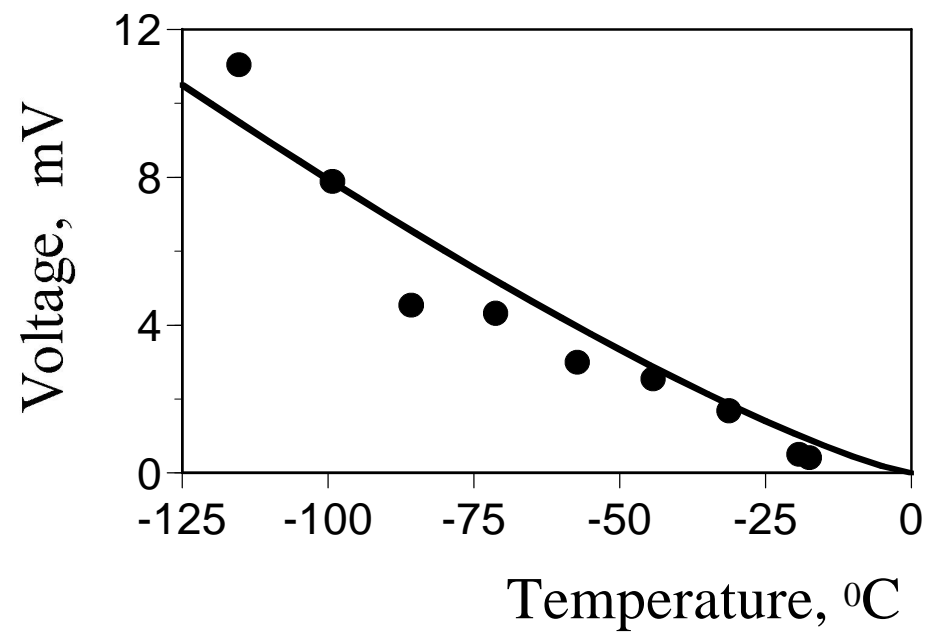

Fig. 5. Peak voltage as a function of difference between the final and initial sample temperatures. Circles are measured data, solid line is calculated (after ${ }^{15}$ ).

To characterize the ability of the GaN film to convert the thermal flow into the electricity, we introduced parameter $P_{f}=d F / d Q$, where $F$ is the electric field, and $Q$ is the heat flow. The model described in Reference ${ }^{15}$ yields

$$
P_{f} \approx \frac{V\left(t_{o}\right)}{Q\left(t_{o}\right) r_{d}}=\frac{\tau_{T} P_{v}}{\rho c_{p}}
$$

and

$$
P_{f}=0.0023 \frac{V m^{2}}{W}
$$

Here $\rho$ is the density and $c_{p}$ is the heat capacity. If the GaN temperature increases by $1{ }^{\circ} \mathrm{C}$ in $1 \mathrm{~s}$, then the generated electric field is $\rho c_{p} P_{f} \approx 60 \mathrm{~V} / \mathrm{cm}$. Unlike the pyroelectric voltage coefficient, this parameter does not depend on heat flow.

As mentioned above, the studied GaN samples had contacts in the plane close to the basal plane. In this case, the collected pyroelectric charge (and the corresponding voltage drop) was 
proportional to a slight $\left(3^{\mathrm{O}}-4^{\mathrm{O}}\right)$ misorientation between the growth direction and the c-axis. Nevertheless, the measured pyroelectric voltage coefficient of $n$-type GaN was on the order of $10^{4} \mathrm{~V} / \mathrm{m}-\mathrm{K}$. As mentioned above, this value is comparable to the values typical for pyroelectric ceramics. ${ }^{13}$ III-Nitride samples having the contacts along the c-axis should allow one to collect more charge and to increase the sensitivity in 20 to 30 times. In addition, GaN exhibit strong pyroelectric effect at temperature above $300{ }^{\circ} \mathrm{C}$, whereas such materials as $\mathrm{LiTaO}_{3}$ and $\mathrm{PbTiO}_{3}$ have the Curie temperature well below $300{ }^{\circ} \mathrm{C}$ and, thus, can not be used for high temperature applications.

\section{Piezoelectric properties of III-Nitrides}

Piezoelectric constants of $\mathrm{GaN}$ are 4-5 times larger than GaAs piezoelectric constants when they are compared using the wurtzite - zinc blende transformation procedure, see Table $1 .{ }^{17}$

Table 1. Piezoelectric constants. (After ${ }^{12}$.) InN constants estimated from ab initio calculations ${ }^{20}$ and using optic phonon frequencies of InN.

\begin{tabular}{|c|c|c|c|c|}
\hline $\mathrm{e}_{\operatorname{lm}}\left(\mathrm{C} / \mathrm{m}^{2}\right)$ & $\mathrm{e}_{33}$ & $\mathrm{e}_{31}$ & $\mathrm{e}_{15}$ & $\mathrm{e}_{14}$ \\
\hline $\begin{array}{l}\text { GaN } \\
\text { (electromechanic } \\
\text { al coefficients) }\end{array}$ & 1 & -0.36 & -0.3 & \\
\hline $\begin{array}{l}\text { GaN } \\
\text { (mobility) }\end{array}$ & 0.44 & -0.22 & $\mid-0.22$ & 0.375 \\
\hline $\begin{array}{l}\text { GaN (from } \\
\text { optical phonons) }\end{array}$ & 0.65 & -0.33 & -0.33 & 0.56 \\
\hline GaN (ab initio) & 0.73 & -0.49 & & \\
\hline $\begin{array}{l}\text { InN (from optical } \\
\text { phonons) }\end{array}$ & 0.43 & -0.22 & -0.22 & 0.37 \\
\hline InN (ab initio) & 0.97 & -0.57 & & \\
\hline $\begin{array}{l}\text { AlN (surface } \\
\text { acoustic waves) }\end{array}$ & 1.55 & -0.58 & \begin{tabular}{|l|}
-0.48 \\
\end{tabular} & \\
\hline AlN (ab initio) & 1.46 & -0.60 & & \\
\hline $\mathrm{SiC}$ & 0.2 & & 0.08 & \\
\hline $\mathrm{ZnO}$ & 1.32 & -0.57 & \begin{tabular}{|l|}
-0.48 \\
\end{tabular} & \\
\hline GaAs & -0.185 & 0.093 & 0.093 & \begin{tabular}{|l|}
-0.16 \\
\end{tabular} \\
\hline
\end{tabular}

This comparison is justified, since GaN has both wurtzite and zinc blende crystal structures. The value of $e_{14}$ for $\mathrm{GaN}$ was extracted from the experimental data on the low field electron mobility using the analysis of the piezoelectric scattering mechanism in the twodimensional electron gas (2DEG) in AlGaN/GaN heterostructures. ${ }^{18}$ The analysis of the optical phonon frequencies of $\mathrm{GaN}$ also yields an estimate of $e_{14}$ of GaN. ${ }^{19}$ A similar estimate of $e_{14}$ for InN implies that the piezoelectric effects in InN are stronger than in GaAs. The wurtzite GaN piezoelectric constants were also estimated from the $\mathrm{GaN}$ electromechanical coupling coefficients. ${ }^{17}$ Finally, the piezoelectric constants of AlN, GaN and InN were predicted using ab initio calculations. ${ }^{20}$ These calculations predict that piezoelectric constants in GaN-based materials are up to ten times larger than in other III-V semiconductors. Currently, it is difficult to determine which set of $\mathrm{GaN}$ piezoelectric constants is the most reliable. However, it is clear that these constants are comparable to those for $\mathrm{AlN}$ and $\mathrm{ZnO}$ and are much larger than for typical $\mathrm{A}_{3} \mathrm{~B}_{5}$ materials, such as GaAs or InP. 
Strongly pronounced piezoelectric properties play a key role in strained GaN-AlGaN and InGaN-GaN multilayer structures. The lattice-mismatch-induced strain generates polarization fields. In a GaN-AlN-GaN semiconductor-insulator-semiconductor (SIS) structure with the growth axis along a (0001) crystallographic direction, the strain-induced electric fields can shift the flat band voltage by $1.5 \mathrm{~V}$ and produce an accumulation region on one side and the depletion region on the other side of the AlN insulator. ${ }^{21}$ As a consequence of the asymmetry in the space charge distribution, the capacitance-voltage $(C-V)$ characteristics and the current-voltage $(I-V)$ characteristics of the symmetrically doped SIS structures become asymmetrical. The degree of the asymmetry depends on strain. This allowed us to develop $C-V$ and $I-V$ characterization techniques for SIS structures and FETs, extract critical thicknesses and demonstrate the gradual elastic strain relaxation process with an increase in strained layer thickness. ${ }^{22,, 24}$

Piezoelectric properties affect the concentration and transport characteristics of 2D electrons confined in the triangular potential well at AlGaN/GaN heterointerface and might lead to accumulation or depletion regions at the heterointerfaces, depending on the polarity of the top surface. $^{25}$ An increase in the 2D-electron sheet carrier density caused by the piezoelectric effects is sometimes referred to as piezoelectric doping. These effects also cause a strain-induced energy band shift at the heterointerface. The surface charge density induced by the piezoelectric effect in AlGaN-GaN Heterostructure Field-Effect Transistors (HFETs) can be on the order of $10^{12}-10^{13} \mathrm{~cm}^{-2} .{ }^{8,26,27}$ These results are very important for developing of a new class high power, high temperature electron devices, such as microwave power amplifiers and power switches.

Piezoelectric properties strongly affect the performance of light emitting devices based on III-N quantum well structures. They can dramatically change the selection rules for the interband transitions in III-Nitride quantum wells and multiple quantum wells (MQWs). Straininduced electric field causes the spatial separation of electron and hole inside the quantum well. ${ }^{28}$ As a result, the optical matrix element for the lowest conduction-band - first heavy hole transition can be dramatically reduced, and the transitions forbidden in strain-free structures can take place. Also, the strain-induced electric field causes a significant reduction of the apparent energy band gap. ${ }^{29}$ This results in a red shift in optical spectra. ${ }^{30}$ The red shift is more pronounced in wider quantum wells and for lower electron-hole concentrations. These effects were predicted 28,31 and confirmed by luminescence emission measurements. ${ }^{30}$ Photoluminescence measurements in InGaN/GaN quantum wells under applied external electric field clearly demonstrated the important role of the built-in field in carrier recombination process. ${ }^{32}$ A strain-induced modulation of optical transitions in quantum structures can be utilized for development of high-speed UV light modulators and fast switching light emitting devices.

In conventional GaAs-based Heterostructure Field Effect Transistors, the 2D-electron gas is induced by doping the wide band gap barrier layer or even the device channel. In AlGaN/GaN based heterostructures, strong piezoelectric effects allow one to induce the 2D-gas without doping. This technique of "piezoelectric doping" avoids the introduction of defects associated with conventional dopants. Electron sheet concentrations as high as $3 \times 10^{13} \mathrm{~cm}^{-2}$ or more can be induced by the piezoelectric effect. ${ }^{8,26,27}$

The elastic strain relaxation and the piezoelectric doping in $\mathrm{Al}_{\mathrm{x}} \mathrm{Ga}_{1-\mathrm{x}} \mathrm{N}-\mathrm{GaN}$ HFETs were studied in References 24,12 . We calculated the $\mathrm{Al}_{\mathrm{x}} \mathrm{Ga}_{1-\mathrm{x}} \mathrm{N} / \mathrm{GaN}$ band structure by solving Poisson's equation with the boundary conditions for the $\mathrm{Al}_{\mathrm{x}} \mathrm{Ga}_{1-\mathrm{x}} \mathrm{N} / \mathrm{GaN}$ interface, which account for the piezoelectric effect:

$$
\varepsilon_{1} \mathrm{~F}_{1}+\mathrm{P}_{1}+\mathrm{P}_{\mathrm{s} 1}=\varepsilon_{2} \mathrm{~F}_{2}+\mathrm{P}_{\mathrm{s} 2}
$$


where $\varepsilon_{1}$ and $\varepsilon_{2}$ are the dielectric constants, and $F_{1}, F_{2}$ are the interface values of the electric field in $\mathrm{Al}_{\mathrm{x}} \mathrm{Ga}_{1-\mathrm{x}} \mathrm{N}$ and $\mathrm{GaN}$, respectively, and $\mathrm{P}_{1}$ is the piezoelectric polarization. The spontaneous polarizations of $\mathrm{Al}_{\mathrm{x}} \mathrm{Ga}_{1-\mathrm{x}} \mathrm{N}$ and $\mathrm{GaN}$ at zero strain, $\mathrm{P}_{\mathrm{s} 1}, \mathrm{P}_{\mathrm{s} 2}$ are accounted for in Eq. (5). For the (0001) growth direction, $\mathrm{P}_{1}$ is given by:

$$
\mathrm{P}_{1}= \pm 2\left(\mathrm{e}_{31}-\mathrm{e}_{33} \mathrm{c}_{31} / \mathrm{c}_{33}\right) \mathrm{u}_{\mathrm{xx}}
$$

The sign in Eq. (6) depends on the polarity of the structure (see a detailed discussion of this issue in ${ }^{33}$ ). Piezoelectric constants, $\mathrm{e}_{31}, \mathrm{e}_{33}$ of $\mathrm{Al}_{\mathrm{x}} \mathrm{Ga}_{1-\mathrm{x}} \mathrm{N}$, were estimated using the linear interpolation between corresponding GaN and AlN piezoelectric constants. The strain in a heterostructure decreases once the layer thickness exceeds the critical thickness. The expression for the deformation energy accounted for the dislocation stress components satisfying the free surface boundary condition and for the dislocation core energy and misfit strain. ${ }^{24}$ A similar approach was developed for superlattices and semiconductor-insulator-semiconductor structures. ${ }^{18}$

In Ref. 24, the sheet electron density was calculated assuming that the spontaneous polarization at zero strain is negligible. In Ref. 12, the calculation of $n_{s}$ was repeated using the spontaneous polarization values estimated in Ref. 20. Figures $6 \mathrm{a}, \mathrm{b}$ show the calculated strain and the sheet electron density, $n_{s}$, generated by the piezoelectric doping in $\mathrm{Al}_{\mathrm{x}} \mathrm{Ga}_{1-\mathrm{x}} \mathrm{N} / \mathrm{GaN}$ HFETs as a function of the Al molar fraction. ${ }^{12}$ The calculation of $n_{s}$ (solid lines in Fig. $6 \mathrm{~b}$ ) accounted for the strain relaxation in HFETs with the AlGaN barrier thicknesses from $5 \mathrm{~nm}$ to 30 nm (Fig. 6 a). Dashed lines in Fig. 6 b correspond to fully strained structures. Also, $n_{s}$ was calculated for the $\mathrm{Al}_{\mathrm{x}} \mathrm{Ga}_{1-\mathrm{x}} \mathrm{N}$ thickness, $L$, equal to the critical thickness, $L_{c}$, for a given $\mathrm{Al}$ concentration (see thick solid line in Fig. 6 b).

The results show that thinner $\mathrm{Al}_{\mathrm{x}} \mathrm{Ga}_{1-\mathrm{x}} \mathrm{N}$ layers with larger $\mathrm{Al}$ content should yield a higher piezoelectric doping. For example, the maximum piezoelectric doping for an unrelaxed heterostructure with $L=L_{c}=5 \mathrm{~nm}$ is $n_{s} \sim 3 \times 10^{13} \mathrm{~cm}^{-2}$, which corresponds to $x \sim 0.6$. This is three times larger than the sheet electron density for $L=L_{c}=20 \mathrm{~nm}$ and $x=0.2$.

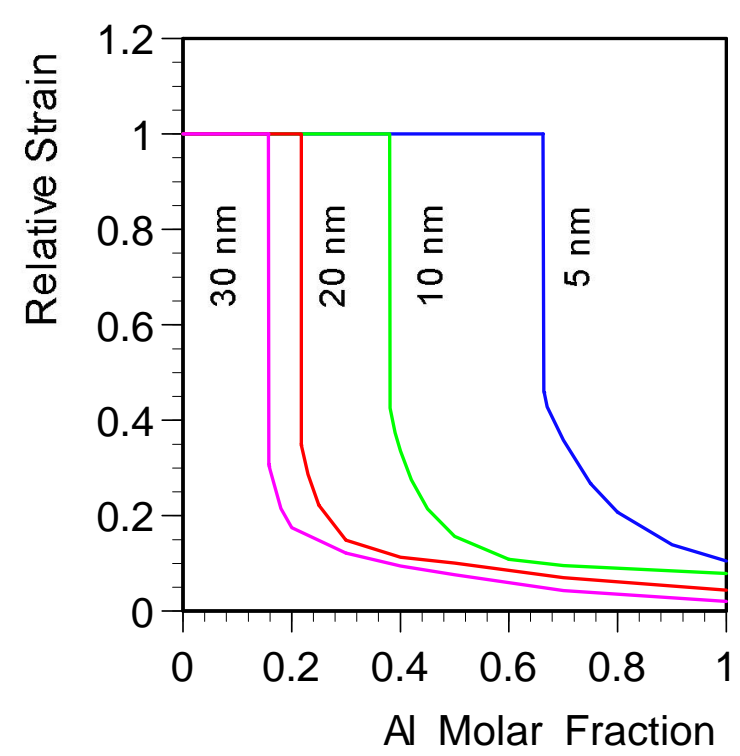

Fig. 6 a. Calculated strain as a function of $\mathrm{Al}$ molar fraction for various $\mathrm{Al}_{\mathrm{x}} \mathrm{Ga}_{1-\mathrm{x}} \mathrm{N}$ layer thicknesses equal to $5 \mathrm{~nm}, 10 \mathrm{~nm}, 20 \mathrm{~nm}$ and $30 \mathrm{~nm}$.

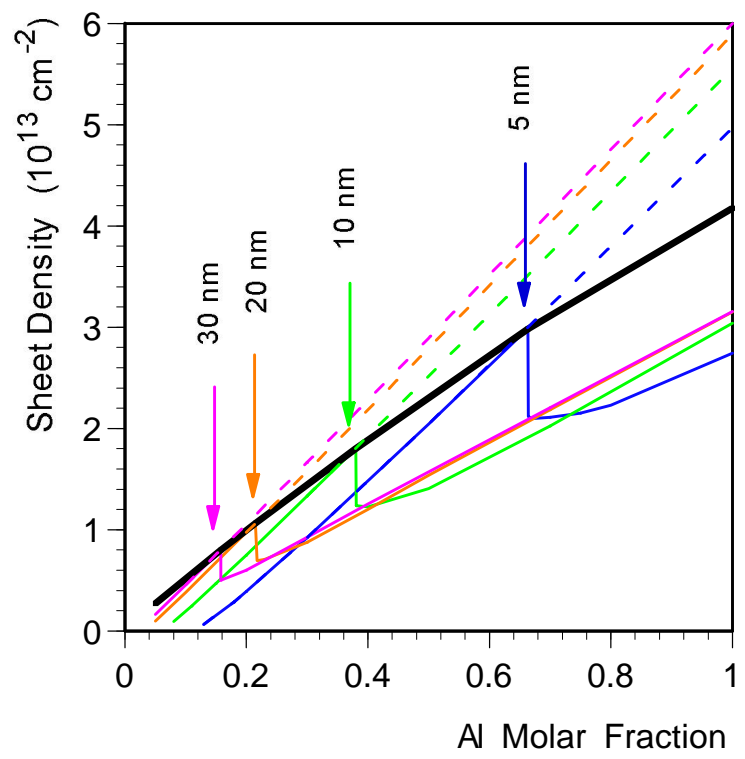

Fig. $6 \mathrm{~b}$. The $n_{s}$ induced by polarization in $\mathrm{Al}_{\mathrm{x}} \mathrm{Ga}_{1-\mathrm{x}} \mathrm{N}-\mathrm{GaN}$ heterostructures for various barrier thicknesses. Arrows show the onset of strain relaxation. Thick solid line shows $n_{s}$ corresponding to critical thicknesses of AlGaN. ${ }^{12}$ 
We also measured the $\mathrm{C}-\mathrm{V}$ characteristics of $\mathrm{Al}_{0.25} \mathrm{Ga}_{0.75} \mathrm{~N} / \mathrm{GaN}$ heterostructures with thicknesses of $\mathrm{Al}_{0.25} \mathrm{Ga}_{0.75} \mathrm{~N}$ ranging from $10 \mathrm{~nm}$ to $100 \mathrm{~nm}$ using standard mercury probe technique. We calculated the C-V characteristics accounting for strain by using Eq. (6) with strain $u_{x x}$ adjusted to fit the experimental data (see Fig. 7). A similar approach was used in order to determine the elastic strain relaxation in GaN-AlN-GaN SIS structures. ${ }^{22,23}$ Figure 6 clearly shows that an increase in $\mathrm{Al}$ mole fraction in the $\mathrm{AlGaN}$ barrier leads to a higher built-in strain and piezoelectric doping of strained $\mathrm{AlGaN} / \mathrm{GaN}$ heterostructures. A high value of $n_{s}$ that can be achieved using the piezoelectric doping points to a high potential of piezoelectric doping as a possible substitute (or an important addition) to conventional impurity doping. The critical thickness of the $\mathrm{Al}_{\mathrm{x}} \mathrm{Ga}_{1-\mathrm{x}} \mathrm{N}$ barrier decreases with an increase in the $\mathrm{Al}$ molar fraction. This, in turn, reduces the depletion charge in the fully strained AlGaN barrier layer. Also, for $x>0.4$, $\mathrm{Al}_{\mathrm{x}} \mathrm{Ga}_{1-\mathrm{x}} \mathrm{N}$ usually becomes insulating. Therefore, in high-quality fully strained AlGaN/GaN heterostructures with high aluminum content, the dominant contributions to the electron gas sheet density should come from the piezoelectric doping as well as from the GaN channel doping. 11,34

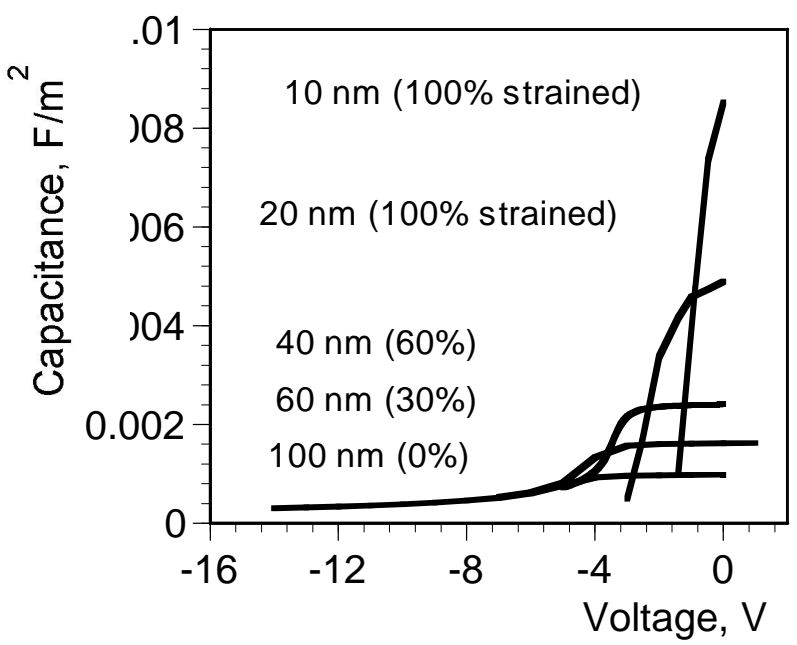

Fig. 7. Measured and calculated (solid lines) capacitance-voltage characteristics of $\mathrm{Al}_{0.25} \mathrm{Ga}_{0.75}$ $\mathrm{N} / \mathrm{GaN}$ structures for different $\mathrm{Al}_{0.25} \mathrm{Ga}_{.0 .25} \mathrm{~N}$ layer thicknesses. Curve 1 is for $10 \mathrm{~nm}$, curve 2 is for $20 \mathrm{~nm}$, curve 3 is for $40 \mathrm{~nm}$, curve 4 is for $60 \mathrm{~nm}$, and 5 is for $100 \mathrm{~nm}$. The structures 1,2 are fully strained, 3, 4 and 5 correspond to elastic strain relaxation of $40 \%, 70 \%$ and $100 \%$. Donor concentrations are $N_{d}=5 \times 10^{17} \mathrm{~cm}^{-3}$ and $10^{17} \mathrm{~cm}^{-3}$ in $\mathrm{Al}_{0.25} \mathrm{Ga}_{0.75} \mathrm{~N}$ and GaN layers, respectively. (After ${ }^{24}$ )

The results obtained by other groups confirm the importance of the piezoelectric doping. Maeda et al. ${ }^{35}$ calculated the maximum two-dimensional electron gas density in $\mathrm{Al}_{\mathrm{x}} \mathrm{Ga}_{1-\mathrm{x}} \mathrm{N} / \mathrm{GaN}$ HFETs by self-consistently solving Schrodinger's and Poisson's equations and accounting for the piezoelectric effect. The maximum 2D-electron gas density was defined as a critical density at which electrons start to spill over into the $\mathrm{Al}_{\mathrm{x}} \mathrm{Ga}_{1-\mathrm{x}} \mathrm{N}$ barrier. The calculated 2D-electron density was in the $2 \times 10^{12} \mathrm{~cm}^{-2}-1.2 \times 10^{13} \mathrm{~cm}^{-2}$ range, depending on the Al molar fraction in the barrier layer, which varied in the range $0.15<x<0.3$, and on the degree of strain relaxation. These calculations assumed that strain relaxation could occur even in HFET with the Al content as low as $x=0.15$ and $\mathrm{Al}_{\mathrm{x}} \mathrm{Ga}_{1-\mathrm{x}} \mathrm{N}$ thickness $15 \mathrm{~nm}$.

\section{Spontaneous Polarization}

First-principle calculations ${ }^{20}$ predict that spontaneous polarization in wurtzite IIINitrides should be quite large. A large spontaneous polarization can cause a large internal 
electric field and strongly affect the band structure. However, the role of the spontaneous polarization depends on the mechanism of the screening of the internal electric field in the bulk of the pyroelectric material and/or at the heterointerfaces. In equilibrium, i.e. without strain or a heat flow, pyroelectric charges are usually compensated by either ambient charges or internal charges forming an equal and opposite dipole moment. An average (macroscopic) internal electric field in heterostructures and superlattices is usually compensated. ${ }^{36}$

In principle, an internal electric field caused by the pyroelectric charge might generate free electrons and holes even in an insulator due to band bending. However, inversion domains with polarization pointing in opposite directions might cancel the macroscopic electric field and reduce an overall energy of the structure (for the experimental evidence of inversion domains in $\mathrm{GaN}$, see ${ }^{37}$ ). The analysis of the piezoresistive measurements in GaN-AlN-GaN SIS structures also provided evidence for the existence of the inversion domains in III-Nitrides. ${ }^{38}$

Another important screening mechanism is linked to the existence of a large number of surface and interface states in pyroelectrics. If the surface state density equals to $P_{s} / q$, the charges trapped in surface and/or interface states can cancel the spontaneous polarization, making an internal electric field vanish inside the film. Indeed, internal electric fields in pyroelectric materials are usually much smaller than predicted by the theory, which does not account for surface and interface states. ${ }^{39},{ }^{40}$ Attempts to find even a trace of an internal electric field at the surfaces of a number of ferroelectrics did not yield any definite results. ${ }^{39}$ The maximum value of a spontaneous polarization charge, $\sim 10^{14} \mathrm{~cm}^{-2}$, observed for all known ferroelectrics is equal to a maximum value of a density of slow and fast surface states in these materials. ${ }^{39}$ The effect of the current-voltage characteristic collapse in some AlGaN-GaN heterostructures indicates the existence of a large number of interface states ${ }^{41}{ }^{42}$, at least in some samples

The measurements of the current-voltage characteristics of GaN-AIN-GaN SIS structures seem to show that the effect of the spontaneous polarization is smaller than that of the polarization induced by the piezoelectric effect. ${ }^{23}$ Hence, further theoretical and experimental studies are needed in order to elucidate a possible role played by spontaneous polarization in GaN-based structures.

\section{Conclusions}

GaN-based materials exhibit strong piezoelectric and pyroelectric effects. Both primary and secondary pyroelectric effects have been observed in GaN based structures. These effects are a combination of a fast response to an initial thermal flow and a slower response to heat dissipation or accumulation. The extracted pyroelectric voltage coefficients of GaN are comparable to those of the pyroelectric ceramics such as PZT or $\mathrm{BaTiO}_{3}$. Also, GaN-based pyroelectric sensors should be capable of operating at very high temperatures, and, hence, have an advantage compared to more traditional pyroelectric sensors.

Piezoelectric effects in GaN and related materials can be used both for piezoelectric sensors and for "energy gap engineering" and "barrier engineering" in GaN-based transistors and other electronic and optoelectronic devices.

The demonstrated piezoresistive effect in GaN/AlN multilayer structures is 2 to 4 times larger than in SiC. ${ }^{38}$ Hence, GaN-based structures can be used in pressure transducers and other electromechanical sensing elements.

However, all these applications are still emerging, and further theoretical and experimental studies are needed. A definite set of piezoelectric constants for GaN-based materials has to be established. The transient response of piezoelectric charge should be investigated in more detail. The role of spontaneous polarization, the role of surface and interface states, and of the inversion domains must be ascertained. The piezoelectric doping 
should be optimized. Further detailed theoretical and experimental studies of strain and dislocations in GaN-based structures are needed in order to better understand the observed stressrelated effects and use these effects for the design of novel piezoelectronic and pyroelectronic devices.

New piezoelectronic and pyroelectronic device concepts will certainly emerge to take a full advantage of the unique properties of GaN-based materials. All in all, the recent research of wide band gap semiconductors with wurtzite crystal structure has opened a new dimension in semiconductor physics and device research. And the work has just begun.

\section{Acknowledgment}

The Office of Naval Research has supported this work (Project Monitors Dr. Colin Wood and Dr. John Zolper).

\section{References}

1 S. Nakamura, M. Senoh, S. Nagahama, N. Iwasa, T. Yamada, T. Matsushita, H. Kiyoku, Y. Sugimoto, Jpn. J. Appl. Phys., Part 2 (Letters), 35, 1B, L74-76 (1996). .

2

S. Nakamura, T. Mukai, and M. Senoh, J. Appl. Phys, 76 (12), 8189 (1994).

M. Razeghi, and A. Rogalski, J. Appl. Phys., 79(10), 7433 (1996).

M. S. Shur, and M. A. Khan, (1997 a). MRS Bulletin. 22, No. 2, 44-50.

J. Burm, K. Chu, W. J. Schaff, L. F. Eastman, M. A. Khan, Q. Chen, J. W. Yang, and

M. S. Shur, IEEE Electron Device Letters 18, No. 4, 141 (1997).

B. J. Thibeault, B. P. Keller, Y. F. Wu, P. Fini, U. K. Mishra, C. Nguyen, N. Nguyen, and M. Le, High Performance and Large Area Flip-Chip Bonded AlGaN/GaN MODFET. (IEDM-97 Technical Digest, IEEE, San Francisco, 1997).

S. C. Binari, J. M. Redwing, G. Kelner, and W. Kruppa, Electronics Letters 33 (3), 242 (1997).

R. Gaska, J. Yang, A. Osinsky, A. D. Bykhovski, and M. S. Shur, Appl. Phys. Lett. 71 (25), 3673 (1997).

R. Gaska, Q. Chen, J. Yang, A. Osinsky, M. A. Khan, and M. S. Shur, IEEE Electron Device Letters. 18, No. 10, 492 (1997).

R. Gaska, J. Yang, A. Osinsky, M. A. Khan, and M. S. Shur, Novel High Power AlGaN/GaN HFETs on SiC substrates, (IEDM-97 Technical Digest, 1997), pp. 565-568. M. S. Shur, and M. A. Khan, Physica Scripta, T69, 103 (1997).

M. S. Shur, A. D. Bykhovski, R. Gaska, and M. A. Khan, GaN-based Pyroelectronics and Piezoelectronics, in Semiconductor Homo- and Hetero-Device Structures, M.

Francombe and C. E. C. Wood, Editors, Academic press, to be published. J. Fraden, Handbook of Modern Sensors, (Springer, New York, 1996), p. 536. M. A. Khan, J. N. Kuznia, J. M. Van Hove, Olson, D. T., Krishnankutty, S., and Kolbas, R. M. Appl. Phys. Lett. 58 (5), 526 (1991).

A. D. Bykhovski, V. V. Kaminski, M. S. Shur, Q. C. Chen, and M. A. Khan, Appl. Phys. Lett. 69, 3254 (1996).

Y. Bayazitolu, and M. N. Ozisik, "Elements of Heat Transfer”, (McGraw-Hill Book Company, New York, 1988), pp. 18-22.

A. D. Bykhovski, B. L. Gelmont, and M. S. Shur, J. Appl. Phys. 81 (9), 6332 (1997).

M. S. Shur, B. Gelmont, and A. Khan, J. Electronic Materials 25, 777 (1996).

M. A. Littlejohn, J. R. Hauser, and T. H. Glisson, Appl. Phys. Lett. 26 (11), 625 (1975).

F. Bernardini, V. Fiorentini, and D. Vanderbilt, Phys. Rev. B 56(16), 10024 (1997). 
A. Bykhovski, B. Gelmont, and M. S. Shur, J. Appl. Phys. 74(11), 6734 (1993).

A. Bykhovski, B. Gelmont, M. S. Shur, and A. Khan, Institute of Physics Conference Series Number 137, 691 (1994).

A. Bykhovski, B. Gelmont, M. S. Shur, and A. Khan, J. Appl. Phys. 77(4), 1616 (1995).

A. D. Bykhovski, R. Gaska, and M. S. Shur, Appl. Phys. Lett. 73 (24), 3577 (1998).

R. Gaska, J. Yang, A. D. Bykhovski, M. S. Shur, V. V. Kaminski, S. M. Soloviev, Applied Physics Letters 72 (1), 64-66 (1998).

P. M. Asbeck, E. T. Yu, S. S. Lau, G. J. Sullivan, J. Van Hove, and J. M. Redwing, Electron. Lett. 33, 1230 (1997).

E. T. Yu, G. J. Sullivan, P. M. Asbeck, C. D. Wang, D. Qiao, and S. S. Lau, Appl. Phys. Lett. 71 (19), 2794 (1997).

J. Wang, J. B. Jeon, Yu. M. Sirenko, and K. W. Kim, Photonics Technol. Lett. 9 (6), 728 (1997).

G. Martin, A. Botchkarev, A. Rockett, H. Morkoç, Appl. Phys. Lett. 68(18), 2541 (1996).

A. Hangleiter, Jin Seo Im, H. Kollmer, S. Heppel, J. Off, F. Scholz, MRS

Internet J. Nitride Semicond. Res. 3, 15 (1998).

C. J. Sun, M. Z. Anwar, Q. Chen, J. W. Yang, M. A. Khan, M. S. Shur, A. D.

Bykhovski, S. L. Weber, M. Smith, J. Y. Lin, and H. X. Xiang, Appl. Phys. Lett. 70, 2978 (1997).

T. Takeuchi, C. Wetzel, S. Yamaguchi, H. Sakai, H. Amano, I. Akasaki, Y. Kaneko, S. Nakagawa, Y. Yamaoka, and N. N. Yamada, Appl. Phys. Lett. 73 (12), 1691 (1998).

E. S. Hellman, MRS Internet J. Nitride Semicond. Res. 3, 11 (1998).

M. S. Shur, and M. A. Khan, MRS Bulletin. 22, No. 2, 44-50 (1997).

Maeda, N., Nishida, T., Kobayashi, N., and Tomizawa, M. (1998). Appl. Phys. Lett. 7 3(13), 1856-1858.

E. L. Ivchenko, P. E. Pikus, Superlattices and other heterostructures: symmetry and optical phenomena (Springer Verlag, Berlin, New York 1995).

L. T. Romano, J. E. Northrup, and M. A. O’Keefe, Applied Physics Letters. 69(16), 2394-2396 (1996).

R. Gaska, J. Yang, A. D. Bykhovski, M. S. Shur, V. V. Kaminski, S. M. Soloviev, Appl. Phys. Lett. 71(26), 3817 (1997).

V. M. Fridkin, Ferroelectrics-Semiconductors (Nauka, Moscow, 1976), p. 90.

B. V. Selyuk, Ferroelectrics. 6, $37-40$ (1973).

M. A. Khan, M. S. Shur, Q. C. Chen, and J. N. Kuznia, Electronics Letters 30, No. 25, 2175-2176 (1994).

M. A. Khan, M. S. Shur, Q. C. Chen, J. N. Kuznia, and C. J. Sun, Electronics Letters

31, 398-400 (1995). 